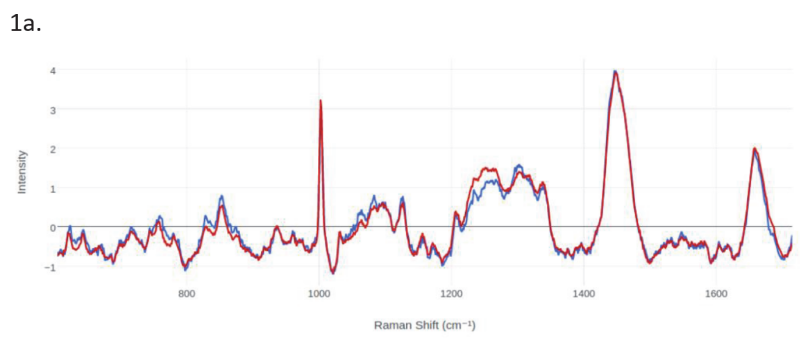

$1 b$.

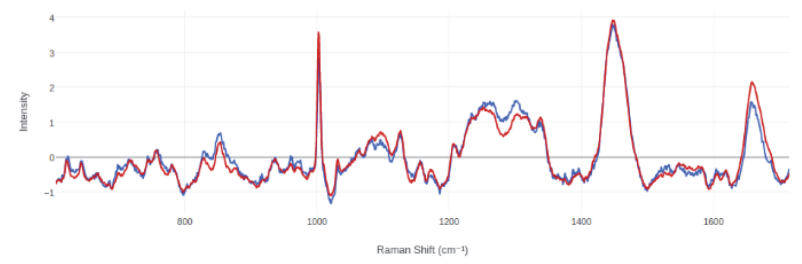

Abstract P157 Figure 1 Self-organising map discriminant index of healing (blue) vs. non-healing (red) in UC (a) and CD (b) demonstrating extracted spectral feautres

UC of $93.9 \%, 99.2 \%, 99.3 \%, 93.6 \%$ and $96.4 \%$ and $93.5 \%$, $98.0 \%, 98.1 \%, 93.1 \%$ and $95.6 \%$ in CD respectively.

1 a.

$1 b$.

Conclusions We have demonstrated that Raman Spectroscopy can accurately differentiate $\mathrm{MH}$ from active inflammation in UC and CD and might be a future tool to direct precise therapeutic management in IBD.

\section{P158 RAMAN SPECTROSCOPY DEMONSTRATES BIOMOLECULAR CHANGES AND PREDICTS RESPONSE TO BIOLOGICAL THERAPY IN INFLAMMATORY BOWEL DISEASE}

${ }^{1}$ Samuel Smith*, ${ }^{2}$ Carl Banbury, ${ }^{3}$ Davide Zardo, ${ }^{1}$ Rosanna Cannatelli, ${ }^{1}$ Olga Nardone, 1,4Uday Shivaji, 1,3,4 Subrata Ghosh, ${ }^{2}$ Pola Goldberg Oppenheimer, 1,3,4 Marietta lacucci. ${ }^{1}$ Institute of Translational Medicine, University Of Birmingham, UK; ${ }^{2}$ Chemical Engineering, University of Birmingham, UK; ${ }^{3}$ University Hospitals Birmingham NHS Foundation Trust, UK; ${ }^{4}$ National Institute for Health Research (NIHR) Birmingham Biomedical Research Centre, UK

\subsection{6/gutjnl-2020-bsgcampus.233}

Introduction Biological therapy in the management of IBD is increasing however, response rates remain modest. Raman Spectroscopy describes the scattering of inelastic light giving spectra that is highly specific for individual molecules revealing tissue biochemistry. Our aim was to establish spectral changes in IBD following biological and whether Raman Spectroscopy can predict response to biological therapy.

Methods IBD patients who underwent endoscopic assessment pre- and 12 weeks post-biological therapy were recruited. Biopsies were taken for ex vivo Raman Spectroscopy analysis alongside biopsies for histological analysis. Response to treatment was defined when both a reduction in the endoscopic score of activity (UCEIS for UC and SES-CD for CD) and histological healing (defined as Nancy $(0-1)$ in UC and modified Riley (0) in CD) was present. For spectral analysis we used artificial neural networks and a supervised learning model to demonstrate spectral differences and build predictive

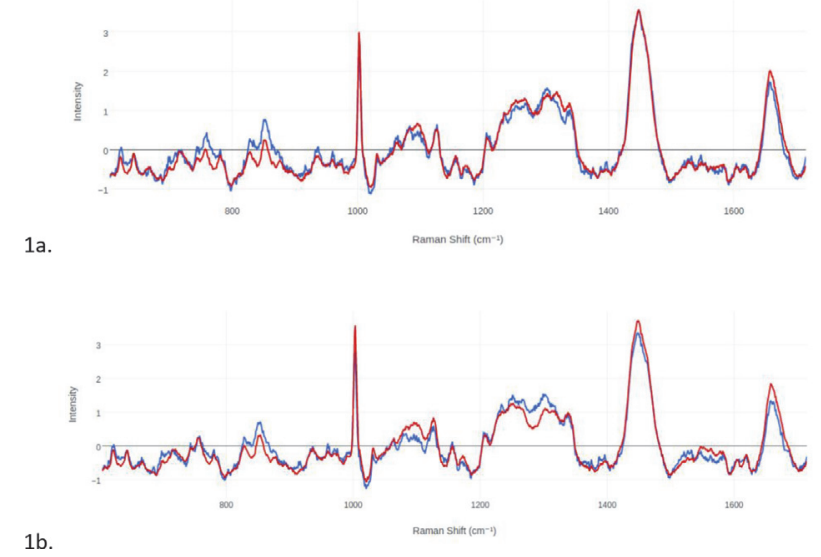

Abstract P158 Figure 1 Self-organising map discriminant index of pre-and post-biological spectra of UC (a) and CD (b) demonstrating extracted spectral features

modelling, based on an 80:20\% (network training: network testing) split of the data.

Results A total of 1800 Raman Spectra (18 patients-7 UC/11 $\mathrm{CD})$ were analysed. Using data projection, there is clear separation between responder ( $3 \mathrm{UC}$ and $3 \mathrm{CD}$ ) and non-responders (4 UC and $8 \mathrm{CD}$ ). The key spectral differences between pre- vs. post-biologic in responders are demonstrated using feature extraction (figure $1 \mathrm{a} \& 1 \mathrm{~b}$ ). There was an increase at $1302 \mathrm{~cm}^{-1}$ after biological therapy and when healing was achieved, which may indicate a potential biomarker of healing.

When comparing the pre-biological spectra, a machine learning algorithm is able to differentiate between responders from non-responders with a sensitivity, specificity, NPV and accuracy of $100.0 \%$ (95\% CI 93.5-100.0), 92.3\% (95\% CI 83.0-97.5), $100.0 \%$ and $95.8 \%$ (95\% CI 90.5-98.6) respectively in UC and CD.

Conclusion We have demonstrated changes in response to biological therapy and a potential biomarker for mucosal healing using Raman Spectroscopy, and can differentiate responders from non-responders in IBD. Using this modelling there is a potential to predict response to biological therapy, however prospective prediction will not need to take place before clinical application. To our knowledge, this is the first study demonstrating these changes.

\section{P159 IMPACT OF FAECAL INCONTINENCE ON HEALTH RELATED QUALITY OF LIFE IN INFLAMMATORY BOWEL DISEASE PATIENTS}

${ }^{1,2}$ Duminda Subasinghe*${ }^{*}{ }^{3}$ Nawarathna Mudiyanselage Meththananda Nawarathna, ${ }^{1,2}$ Dharmabandhu Nandadeva Samarasekera. ${ }^{1}$ Department of Surgery, University of Colombo, Colombo, Sri Lanka; '2University Surgical unit, The National Hospital of Sri Lanka, Colombo, Sri Lanka; ${ }^{3}$ Department of Medical Gastroenterology, The National Hospital of Sri Lanka, Colombo, Sri Lanka

\subsection{6/gutjnl-2020-bsgcampus.234}

Introduction To analyze the frequency and severity of faecal incontinence (FI) and its effect on the quality of life (QOL) in inflammatory bowel disease (IBD) patients.

Methods All patients who attended surgical and medical gastroenterology outpatient clinics in a tertiary care center with an established diagnosis of either ulcerative colitis (UC) or Crohn's disease (CD) over a period of 10 months 
were included in this study. Before enrolment into the study, the patients were explained about the study and informed consent was obtained. The patients with unidentified colitis were excluded. The data on demographics, disease characteristics, FI (Vaizey score), and quality of life (IBD-Q) were collected. Data were analyzed using SPSS version 21.

Results There were 184 patients (women $=101,54.9 \%$; UC $=153,83.2 \%$ ) with a female preponderance for UC (male/ female ratio $=1: 1.5$ ) and a male preponderance for CD (male/female $=2: 1$ ). Forty-eight $(26 \%)$ patients reported symptoms of FI. Among the patients with FI, 70.8\% were women $(\mathrm{n}=34)$ and $29.2 \%$ were men $(\mathrm{n}=14)$ with an average age of 52.7 years (range, 20-78 years). Average age of onset of FI was 48.6 (range, 22-74) years. Ten percent (n $=5$ ) reported regular FI. Incontinence to flatus was seen in $33.3 \%(\mathrm{n}=16)$, to liquid faeces in $56.2 \%(\mathrm{n}=27)$, to solid faeces in $6.2 \%(n=3)$ and to all three in $4.1 \%(n=2)$. Twenty-one percent $(\mathrm{n}=10)$ complained of disruption of their physical and social activity. There was no association between FI and type of IBD. Significant associations were found between FI and age $(\mathrm{P}=0.005)$ and gender $(\mathrm{P}<$ 0.001). QOL in our cohort of patients was significantly affected by FI.

Conclusions In our study, nearly a quarter of patients reported FI. There was a significant correlation between FI and QOL. Therefore, enquiring about FI in IBD patients can lead to identification of this debilitating condition. This will enable early referral for continence care in this group of patients.

\section{P160 PRE-BIOLOGIC SCREENING IN A HIGH RISK AREA: ARE WE ADHERING TO GUIDELINES?}

Eilis Kempley, Rajan Pooni, Cheh Kuan Tai*, Noor Jawad. Newham University Hospital, London, UK

\subsection{6/gutjnl-2020-bsgcampus.235}

Introduction Newham University Hospital, Bart's NHS Trust, serves the London Borough of Newham which had the highest incidence of tuberculosis in the UK at 78.0 per 100,000 in 2014. Newham also has the highest average annual rate of new reported acute hepatitis $B$ infection in the UK. There have been clear guidelines on pre-biologic screening for opportunistic infections since 2014. Our aim is to assess whether patients who are on biologic therapy have been appropriately screened prior to initiation of biologic therapy.

Methods A retrospective review of all IBD patients on the biologic database was performed in November 2019. Patients who were initiated on biologics prior to the publication of guidelines in June 2014 were excluded.

Results The total number of patients was 63. 31 patients (49.2\%) had latent tuberculosis testing with Interferon-gamma release assay (IGRA) testing and 2 were positive. Screening with Chest XR (CXR) was better with 58.9\% concordance. 36 patients had normal CXRs and 1 had an appearance of a granuloma.

In comparison, viral screening had higher completion rates. Hepatitis B Surface Antigen (HbsAg) was sent in 53 patients $(84 \%)$ and all were negative. Hepatitis B Core Antibody $(\mathrm{HbcAb})$ was sent in 23 patients $(36.5 \%)$ and 1 was positive.

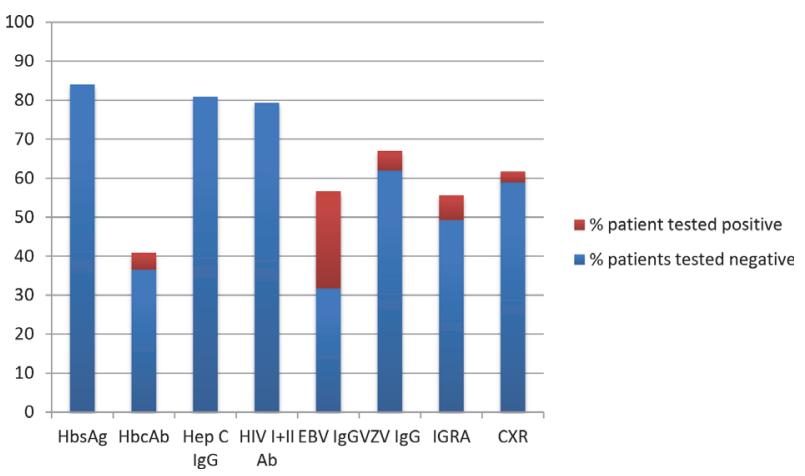

Abstract P160 Figure 1 Percentage of patients who received respective pre-biologic test

1 patient was $\mathrm{HbcAb}$ positive but $\mathrm{HbsAg}$ negative. In terms of Hepatitis C, 51 patients (80.9\%) had Hepatitis C IgG sent and all were negative. All 50 patients (79.3\%) who were tested for Human immunodeficiency virus (HIV I+II antibody) were negative. Ebstein Bar Virus IgG was sent in 20 patients (31.7\%), out of which, 15 were negative. Varicella-Zoster Virus IgG was sent in 39 patients $(61.9 \%)$ and 2 were positive.

An infection history was not taken, for either bacterial, fungal or viral infections and Bacille Calmette-Guerin vaccination status was not documented. No documentation was present regarding measles status. Routine vaccination status was not confirmed for diphtheria, poliomyelitis, pertussis, tetanus or Human Papilloma Virus. Prior to initiation of immunomodulation, vaccination was not considered for pneumococcal or influenza infections.

Conclusions Despite suboptimal pre biologics screening in this high risk region of East London for Tuberculosis and Hepatitis $\mathrm{B}$, no cases of reactivation of either Tuberculosis or Hepatitis $\mathrm{B}$ have been identified to date. The results suggest that clinicans are requesting some tests but not all. Following on from these results, we will be streamlining the process for ensuring all tests are performed prior to biologic initiation with a checklist proforma for the patients notes and on our biologics database for all prescribing gastroenterologists, as per ECCO guidelines.

\section{P161 IKK $\alpha$ AS A POTENTIAL THERAPEUTIC TARGET FOR THE PREVENTION OF INFLAMMATORY BOWEL DISEASE}

${ }^{1}$ Joseph Tang*, ${ }^{1}$ Stamatia Papoutsopoulou, ${ }^{2}$ Andrew Paul, ${ }^{2}$ Professor Simon Mackay, ${ }^{1}$ Carrie Duckworth, ${ }^{1}$ Mark Pritchard. ${ }^{1}$ University Of Liverpool, Liverpool, UK; ${ }^{2}$ University fo Strathclyde, Glasgow, UK

\subsection{6/gutjnl-2020-bsgcampus.236}

Introduction Current treatment options for inflammatory bowel disease (IBD) are primarily designed to suppress an established inflammatory response, but they do not prevent the initiation of the inflammatory cascade. We have previously demonstrated that the NF- $\mathrm{KB}$ signalling pathways play a pivotal role in murine experimental models of IBD. In particular, we showed that $n f \kappa b 2-/-$ mice were protected against LPSinduced cell shedding and DSS-induced colitis compared to wild-type mice. Specifically, our data suggested that NF-кB2 signalling in intestinal epithelial cells played a more important 